\title{
Ultrasound for Critical Care Physicians: Hypotension After a MVA
}

A 25 year old woman was a restrained driver in a rollover motor vehicle accident (MVA) and suffered a C5-C6 fracture-dislocation with spinal cord injury. She was lucid and able to follow commands and could move her upper extremities but not her lower extremities. She was given approximately 6 liters of fluid but required vasopressors to maintain her blood pressure. Initial ECG revealed a normal sinus rhythm without significant ST changes (Figure 1).

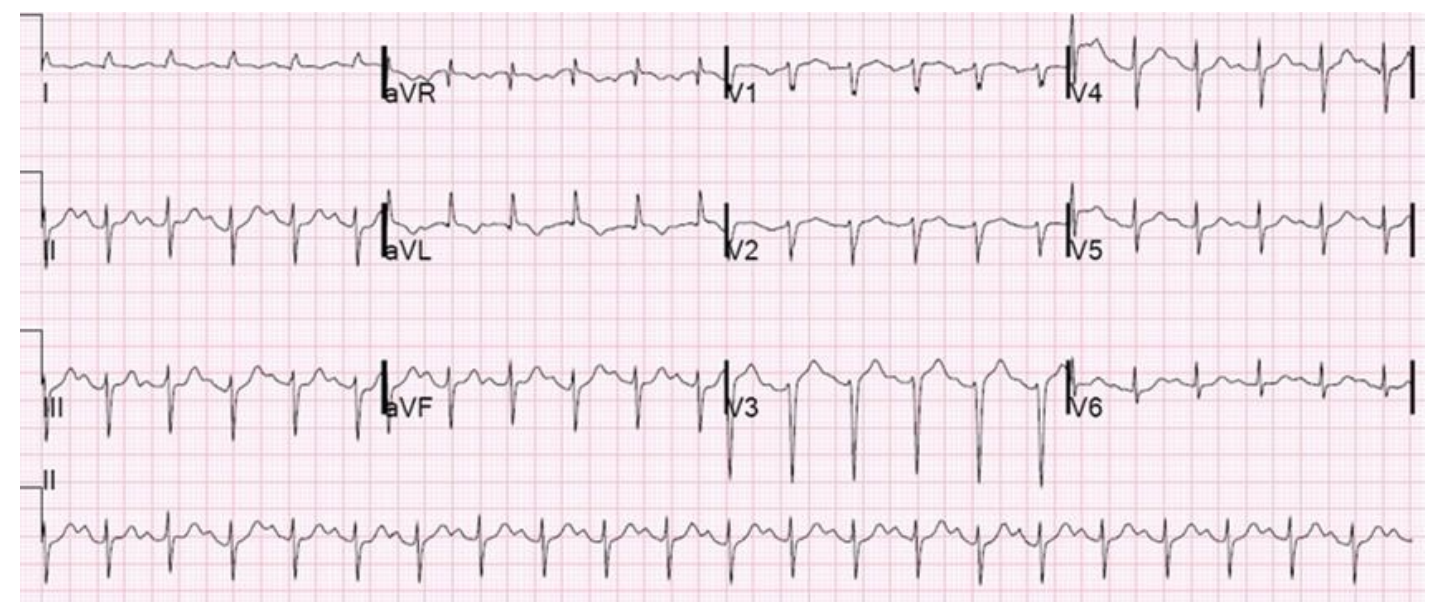

Figure 1. Initial ECG.

Upon initial evaluation her blood pressure was low. Bedside ultrasound of the left anterior second intercostal space revealed a sliding lung sign and a 4 chamber view of her heart was performed (Figure 2).

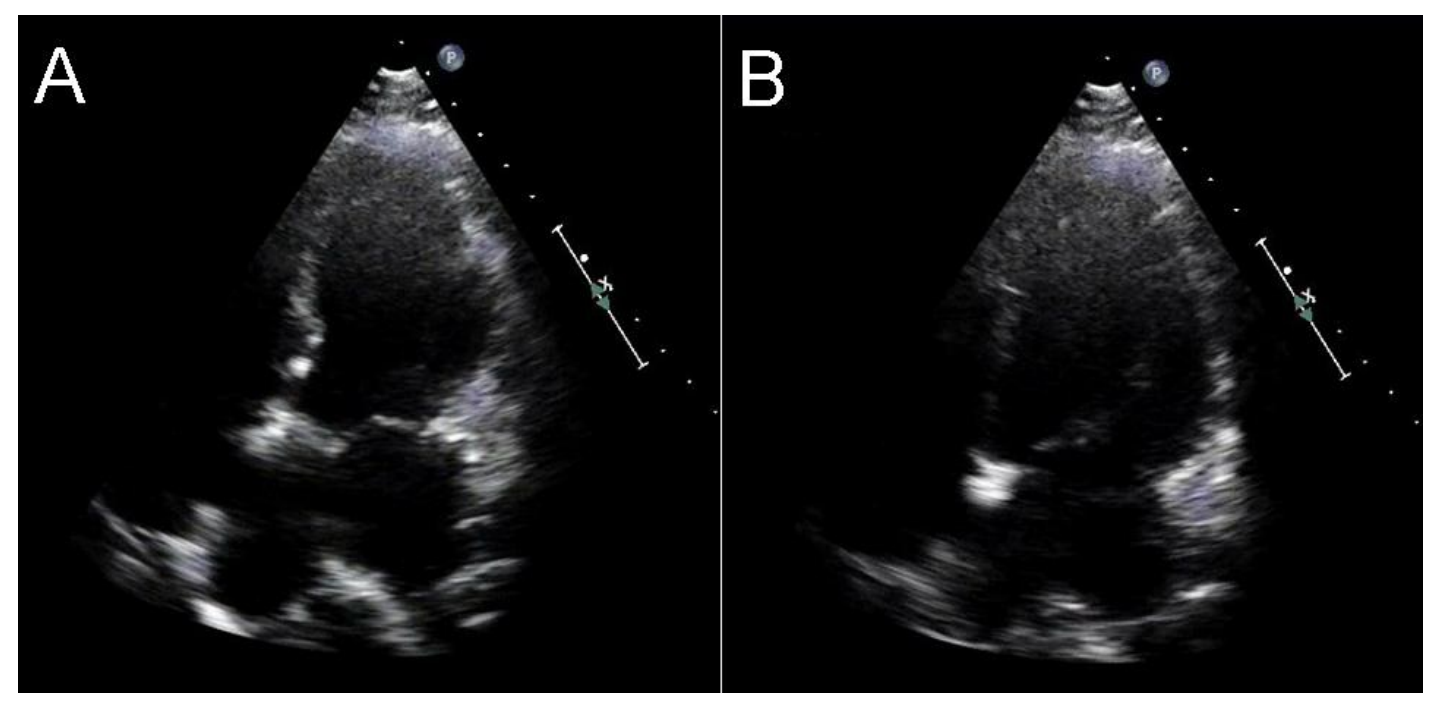

Figure 2. Representative static images from the cardiac ultrasound. Panel A: systole. Panel B: diastole. 
Which of the following is the most likely cause of her hypotension?

1. Blunt cardiac injury

2. Intravascular volume depletion

3. Neurogenic stunned myocardium

4. Pericardial tamponade

5. Pneumothorax 


\section{Correct! \\ 3. Neurogenic stunned myocardium}

The cardiac ultrasound shows left ventricular apical/lateral hypokinesia and an ejection fraction of approximately 30-35\%. A high index of suspicion should be maintained for both blunt cardiac injury and pneumothorax. Blunt cardiac chest trauma resulting in myocardial contusion and dysfunction is a possibility but would be an unusual cause of hypotension with her fairly normal ECG. A sliding lung sign is fairly sensitive for excluding pneumothorax (1). In this case, insertion of a left chest tube resulted in no improvement in blood pressure. She had received 6 liters of fluid without improvement in her hypotension. In the absence of hemorrhage, this makes intravascular volume depletion unlikely. Neurogenic shock is a possibility and likely accompanied the cardiac changes in this patient but was not one of the choices.

Neurogenic stunned myocardium has been described in subarachnoid hemorrhage, intracerebral hemorrhage, acute ischemic stroke and head trauma (2). It is thought to be similar to stress-induced cardiomyopathy (Takatsubo's cardiomyopathy) or apical ballooning syndrome. Neurogenic stunned myocardium is thought to be initiated by an injury to the hypothalamus leading to a surge in adrenergic output with a resultant increase in norepinephrine release within the myocardium, however, plasma concentrations remain in the normal range. This leads to myocyte calcium overload and contraction band myonecrosis with resultant systolic heart failure. Myocardial scintigraphy has confirmed normal perfusion with normal MIBI uptake with abnormal MIBG uptake correlating with the regional wall motion abnormalities seen in patients who develop NSM. The resultant functional myocardial sympathetic denervation leads to cardiac injury and release of troponin.

Evan D. Schmitz, MD

Richland, Washington

\section{References}

1. Husain LF, Hagopian L, Wayman D Baker WE, Carmody KA. Sonographic diagnosis of pneumothorax. J Emerg Trauma Shock. 2012;5(1):76-81. [CrossRef] [PubMed]

2. Banki NM, Kopelnik A, Dae MW, et al. Acute neurocardiogenic injury after subarachnoid hemorrhage. Circulation 2005;112(21):3314-9. [CrossRef] [PubMed] 